\title{
The Role of the Player in Video-Game Fictions
}

\author{
Aaron Suduiko*
}

What is the relationship between the player and the avatar of a video game? In this article, I aim to show that Jon Robson and Aaron Meskin's apparently promising, Waltonian analysis of that relationshipnamely, that it consists in the player imagining herself as the avatar-fails to accommodate and explain four central data about video-game storytelling that any such analysis ought to accommodate and explain. These data are, briefly: (1) Many of an avatar's actions are inexplicable if we appeal only to the avatar's beliefs, desires, and knowledge. (2) Video games may have many different kinds and numbers of avatars. (3) Video-game narratives often proceed by the player exploring multiple disjunctive, mutually exclusive possibilities. (4) Video-game narratives sometimes centrally depend on epistemic differences between the player and avatar. After evaluating Robson and Meskin's view, I offer my own positive analysis of player interactivity that provides a motivated and unified explanation of these four data: the player of a video game plays the role of a metaphysically foundational fictional entity that actualizes possible fictional events. I call this entity 'the fictional player'.

Keywords: avatars, narrative, video games, storytelling, fiction, narrative explanation, ontology, fictional grounding, art, aesthetics, possible worlds, Walton.

Suppose you sit down to enjoy a video game. You turn on your television, fire up your PlayStation, and a digital world emerges on the screen. In the middle of that world stands a character; as you press buttons and push sticks on your controller, a characterthe avatar-begins to move and perform various actions.

It is clear enough that, by exerting control over this avatar, you are involving yourself with the avatar and its world in some way. But exactly what kind of involvement is this? What, exactly, is the relationship between a player and an avatar?

In recent years, game theorists have established a wide range of possible answers to this question. Rune Klevjer claims that "[the] relationship between the player and the avatar is a prosthetic relationship; through a process of learning and habituation, the avatar becomes an extension of the player's own body” (Klevjer 2006, 10). James New-

* This article is largely derived from my undergraduate thesis at Harvard University, completed in 2017. Thanks especially to Richard Moran, my advisor for that thesis. Thanks also to Ned Hall, Sean Kelly, Bernhard Nickel, Alison Simmons, Jack Davis, Rune Klevjer, John R. Sageng, two anonymous reviewers, my undergraduate thesis workshop group, and the community of analysts and gamers at WithATerribleFate.com. 
man claims that "[the] player utilises and embodies the character [i.e. avatar] in the gameworld," where this embodiment is defined by "sets of capabilities, potentials and techniques offered to the player" (Newman 2002). Mark Bell defines an avatar as "any digital representation (graphical or textual), beyond a simple label or name, that has agency (an ability to perform actions) and is controlled by a human agent in real time," and that therefore "function like user-controlled puppets" (Bell 2008, 3). Jesper Juul says that when an avatar does some action in a video game, then the player, "by extension,” does that same action (Juul 2005a, 183).

The wide range of accounts that game theorists have put forth to explain the playeravatar relationship reflects, in part, the fact that the term 'avatar' is itself quite capacious. At first blush, for example, we might describe both the character of Cloud Strife in Final Fantasy VII and a real-world, remote-piloted drone as avatars, despite deep and non-controversial differences between the two cases-for instance, Cloud is a character within a work of fiction, whereas the drone is entirely non-fictional, though both are remotely manipulable entities within a game (Cloud within the game of Final Fantasy VII, and the drone within a war game). (Square 1997.)

Some game theorists have adopted more narrow conceptions of 'avatar' in order to answer questions with a correspondingly narrower scope. Philosophers of aesthetics, for example, have focused on those avatars that function as characters within a fictional story. This is the focus that will occupy us in this article.

When it comes to analyzing the player-avatar relationship specifically in video-game storytelling, one idea put forward by Jon Robson and Aaron Meskin (2016) seems especially promising: they claim that this relationship consists in the player imagining herself as the avatar. As they note in their analysis of player involvement in video game fictions, claiming that the avatar is fictionally the player has two advantages:

1. It tracks the ordinary language that the players of video games use to discuss their activity in those games-e.g., when a player's avatar is $\varphi$-ing, it is apt for the player to say that she, the player, is $\varphi$-ing (Robson and Meskin 2016, 169).

2. It is a conservative extension of Kendall Walton's (1990) theory of fiction as prescriptions to imagine certain propositions (ibid., 170-172).

It is my aim in this article to show that, while it seems attractive to agree with Robson and Meskin that player involvement in the stories of video games consists in the player imagining herself as the avatar, their analysis fails to accommodate four central data about video-game storytelling: (1) the explanations of avatars' actions, (2) the variability of what can constitute an avatar in different video games, (3) the exploratory nature through which players often advance video games' stories, and (4) the differences in the beliefs, desires, and knowledge of avatars and players. I begin with a comment on the scope of stories that I will be considering. Then, in the first half of the article, I show 
why a theory of video-game storytelling ought to accommodate and explain the aforementioned four data, and argue that Robson and Meskin's theory fails to do so. In the second half of the paper, I pivot to offer my own positive account of how a player interacts with video-game stories: namely, the player assumes the role of a fictional, metaphysical agent that grounds the events of a video game's story. While it is beyond the scope of the article to compare this analysis against all present alternatives in the literature, I argue that it warrants further consideration based on its capacity to accommodate and explain all four of the central video-game-storytelling data under consideration in a unified, well-motivated way.

\section{A Note on Scope}

After this section, I will be referring to the storytelling medium I wish to analyze as 'video games'. However, the medium that I have in mind is not coextensive with the huge set of objects that we ordinarily call "video games." Thus it is important to clarify precisely what type of story I am considering, and why I think it merits special consideration.

I am interested in considering video games as a storytelling medium, and in seeing how player involvement adds a new dimension to traditional conceptions of narrative. Therefore, of the broader set of all video games, I am only considering those that convey a narrative. Here, I follow J. David Velleman (2003) in defining 'narrative' as a series of events with a plot, or "the principle of organization by which narrative confers intelligibility on narrated events" (Velleman 2003, 4). So, although Tetris is a video game, I will not be considering it as such here, since the events of the game lack a plot (Pajitnov, 1984). ${ }^{1}$

I am interested in video games as a medium that can represent multiple possible narratives that are causally related to one another, where the person engaging one such narrative has some degree of control over which subsequent narrative is brought about as they engage the medium. So, for example, two different narratives might proceed in a game based on whether or not the avatar - the character that the player controls — chose to $\varphi$ or to $\psi$ following some event $e$ in the game. Notice that there are varieties of interactive, storytelling media other than video games that satisfy this condition: chooseyour-own-adventure novels, most notably, would count as "video games" according to this condition.

It is worth noting that this second condition on my category of 'video game' motivates an application of possible-worlds talk in a way distinct from the popular usage of "fictional worlds" of which Walton is critical. Walton notes that "[fictional] worlds are sometimes impossible and usually incomplete, whereas possible worlds (as normally

1 I acknowledge, though, that some, such as Janet Murray, disagree (Murray 1997, 144). 
construed) are necessarily both possible and complete" (Walton 1990, 64). Let us grant that these are reasons enough for not talking of "fictional worlds" as if they were bona fide possible worlds; all the same, in the context of video games, we can think of a game's distinct narratives as relevantly similar to possible worlds: just as we can distinguish between the actual world and possible worlds that are counterfactually related to it, we can distinguish between the actual narrative of a video game- the narrative that the player brings about and experiences, otherwise known as a playthrough —and the possible narratives that are counterfactually related to it, and that could be the actual narrative if the player made different choices.

Ultimately, I think that it is this possibility structure - the total set of possible narratives in a video game- - that makes this storytelling medium I aim to examine uniquely interesting. Two of the central data about video-game storytelling that I will later examine- the explanatory and exploratory data-essentially refer to the possibility structure. My positive explanation of player interaction with video games, too, essentially depends on the possibility structure to establish the fictional ground of video-game narratives' events.

\section{Robson and Meskin's Analysis of Video Games as Self-Involving Interac- tive Fictions}

Robson and Meskin claim that video games are self-involving interactive fictions ('SIIFs'): "fictions that, in virtue of their interactive nature, are about those who consume them” (Robson and Meskin 2016, 165). In Waltonian language, which Robson and Meskin themselves employ, members of this class of fiction prescribe that the audience members imagine certain propositions about themselves as part of that work itself.

The scope of Robson and Meskin's SIIF concept is strictly wider than what I am referring to as video games. ${ }^{2}$ The self-involving "video games" that they are considering only need to be cases of fiction-i.e. a set of fictional truths, or, equivalently, prescriptions for audience members to imagine certain things (ibid., 166). For our purposes, therefore, I will just be focusing in the first instance on their analysis of SIIFs as it pertains to the relationship between player and avatar in video games that have narratives. (When I discuss the variability datum, I will zoom out to consider a case that Robson and Meskin claim does not involve an avatar.)

2 Robson and Meskin themselves are agnostic regarding whether or not choose-your-own-adventure novels are SIIFs, but they think that they could plausibly be interpreted in this way (Robson and Meskin 2016, 172-173). I will not dwell on that issue since choose-your-own-adventure books are a fringe case for the purposes of this article. 
According to Robson and Meskin, the player of a video game relates to her avatar by imagining that she is identical with that avatar, like a child who imagines that she is Superman (ibid., 169). In virtue of this fictional identity between player and avatar, whatever is fictionally true of the avatar is also fictionally true of the player. Robson and Meskin's language suggests that they believe there are some exceptions to this relation, ${ }^{3}$ but this is the principal model that they present for avatar-player relations.

The major appeal of this theory is that it uses Walton's popular theory of fiction to explain how players interact with the stories of video games in a way that preserves vast swaths of ordinary discourse and ways that players really think about video games. As Robson and Meskin say, it is perfectly natural for a group of people watching someone play a game refer to the game's avatar by the name of the game's player: if Sam and Alex are watching their friend, Susan, play a game in which the avatar is fighting a strong enemy, all of the following locutions would be natural:

- Alex to Sam: "Wow, Susan is fighting a very strong enemy."

- Sam to Susan: "Wow, you are fighting a very strong enemy."

- Susan to Alex: "Wow, I am fighting a very strong enemy.”

It is also fitting that Robson and Meskin discuss their theory using the example of Dark Souls II, a game in which the player is able to determine her avatar's sex, physical features, history, and abilities: in many games, players can and will design their avatars to represent a role that they want to play, which reinforces the idea that players are trying to make things fictionally true of themselves by making things fictionally true of their avatars (FromSoftware 2014).

Despite the appeal of Robson and Meskin's theory, it ultimately fails as an analysis of the player's involvement in the stories of video games. This is because it can neither accommodate nor explain four central data about the storytelling of video games: the explanatory datum, the variability datum, the exploratory datum, and the epistemic datum. By 'data', I mean features of a domain of theorizing that anyone reasonably well acquainted with the domain would recognize at the outset of theorizing; that the data are central to the domain of video-game storytelling means that any theory of video-game storytelling must accommodate and explain those data. ${ }^{4}$ I will examine each of these data in turn.

3 Specifically, the following language: "these decisions not only make certain things fictional concerning her avatar but also - given that her avatar is fictionally her-make many of the same things fictionally true about her" (ibid., 168, emphasis mine).

4 I owe this conception of data to Bengson, Cuneo, and Shafer-Landau (forthcoming). 


\section{The Explanatory Datum}

The explanatory datum about video games is the following: in any given videogame narrative, many of the avatar's actions are inexplicable if we appeal only to the avatar's beliefs, desires, and knowledge. A key type of case in which the explanatory datum obtains is failing-and-repeating: the iterative process of the player finding "success conditions" for proceeding through a video game's narrative.

There are often conditions for "failure" in a video game in the following sense: it is possible for an avatar to die in the course of a video game's narrative, e.g. by failing to kill some particular enemy, at which point the player will have to return to some particular earlier position in the playthrough (often the last point at which the game was saved) and try to find a way to proceed beyond that point without the avatar being killed. Failing-and-repeating is essentially a process of the player learning the various possible outcomes of the game, iterating various commands to the avatar until they find success conditions for proceeding through the narrative. This means that players often use information from their failed attempts in order to ultimately succeed: perhaps the player learns-by-death-of-the-avatar in Skyrim that a large, disproportionately powerful monster lurks behind a hidden but avoidable corner; subsequently, she proceeds by directing her avatar to avoid that corner (Bethesda Game Studios 2011). Suppose the player named her avatar "Icarus": we would surely be licensed to ask, as we would when making sense of a character's actions in any such narrative, "Why did Icarus avoid that corner as he progressed through the area?" The obvious answer seems to require that we cite the monster that previously killed Icarus, but surely Icarus, as he (fictionally) lives and breathes, does not know about that monster. One might instead try to construct some story about how Icarus "had a bad feeling" about the corner, or how he simply avoided it by dumb luck, but these ad hoc explanations willfully avoid the apparent, actual reason why Icarus did not go explore the corner: the player knew about the monster waiting for Icarus.

If the fictional truths about the player of Skyrim are identical with those about Icarus, then this case-and analogous cases involving failing-and-repeating-will be inexplicable within the fiction of video games (ibid.). Suppose we try to appeal to ordinary language about video games here, as Robson and Meskin did when developing their view: I am watching my friend play Skyrim when she has Icarus avoid the corner, and I ask her: "Why did you do that?” (ibid.) The player might respond in our example, “Oh, I explored that corner before, and a monster killed me.” Yet we surely cannot be expected to take this language as a literal account of what is going on in the narrative. Even if players often imagine that they are their avatars, they surely do not play through all video games while imagining that they are beings with the (often narratively implausible) ability to infinitely reincarnate and try out different actions every time they die, à la Bill Murray's character in Groundhog Day (Albert and Ramis 1993). 


\section{The Variability Datum}

The variability datum of video games is the following: video games, even given the constraints I set out in my note on scope, possess ample diversity in the exact makeup of their ontological constituents. In particular, a video game may have just one avatar that the player apparently controls throughout the narrative; a video game may have the player apparently control different or multiple characters throughout the narrative; and a video game may even have the player apparently control some object that is not an avatar (e.g., a racecar). An adequate analysis of video games must be generalizable to the extent that it accommodates this apparent diversity, or else it must furnish us with some explanation of why it need not generalize.

Robson and Meskin's view can handle the variability datum fairly well. In a game where the player can choose which of several characters to use as an avatar, or a game where there are different avatars at different points in the narrative, they can simply say that the player imagines herself as a different character (i.e. as the current avatar) each time a different character becomes the avatar. ${ }^{5}$

The view fares less well, I think, when we consider the variability between games that do and do not have avatars: in particular, it runs the risk of stipulating avatars in games where there are none.

For instance, consider Robson and Meskin's analysis of wipEout, a video game in which the player controls a racing craft rather than an avatar (Psygnosis 1995). Robson and Meskin claim that players engage the fiction by imagining themselves as drivers of the racing craft (Robson and Meskin 2016, 168). They motivate this analysis by appealing to the language players use when discussing the game, saying things like "[I am] moving at a breakneck speed," which they think speaks in favor of it being fictional that the player of the game is the driver of the racing craft.

I agree with Robson and Meskin's assessment that there is no avatar in wipEout-the player controls a vehicle with no driver visually represented-but I worry that their analysis ends up implying that there is an avatar in the game, despite their explicit disavowal of any avatar being present. ${ }^{6}$ Robson and Meskin contend that the difference between a game with an avatar and a game like wipEout is akin to the difference between imagining that one is Superman and imagining that one has Superman's abilities without imagining that one is identical with any particular individual (ibid., 169). But it

5 Thanks to an anonymous reviewer for emphasizing this point to me.

6 The reader might disagree with the claim that there is no avatar in WipEout-maybe there is something like an "implied driver" within the racing craft. I think that, for the sake of argument, it is easier to simply agree with Robson and Meskin's stipulation that there is no avatar in this case; however, even if one thinks that there is an avatar in this case, there are analogous cases in which the absence of an avatar is clearer (see Fire Emblem in "Explaining the Variability Datum”). 
seems to me that, if the player is relating to wipEout by imagining that she is the driver, she is imagining that she is an individual: namely, the unseen pilot inside the racing craft (Psygnosis 1995).

To suppose the player is really imagining things about herself without imagining that she is an avatar within the game is to say that the player is imagining that she, a person outside the world of the game (sitting on a couch, or what have you), is manipulating objects within the game's world. I do not think that Robson and Meskin take the player of wipEout to be having this unusual kind of experience-but if they do think that the player is imagining herself as the pilot within the craft, then I think it is necessary to stipulate that there is an avatar within the racing craft, even though we do not see the avatar (ibid.). Thus Robson and Meskin's analysis yields a false positive: an avatar in a video game without one.

\section{The Exploratory Datum}

The exploratory datum of video games is this: a typical part of the act of playing a video game and consuming its narrative is the act of exploring its possibility structure. To see what this exploration consists in, suppose that you are playing Dishonored and arrive at a confrontation between the avatar, Corvo, and an assassin, Daud (Arkane Studios 2012). When Daud asks Corvo to spare his life, you deliberate and decide to have Corvo kill him. However, you are curious: what would have happened had you instead directed Corvo to spare Daud? Counterfactuals like this in video games are verifiable in that the player can actually go back and see what would have happened in a counterfactual fictional scenario like the one with Corvo and Daud. Because video games allow the player to save her game at certain points, cataloging certain locations in a given playthrough to which she can return at will, it is easy for a player to, after having Corvo kill Daud, load the save file that marks the moment before she reached the Corvo-Daud confrontation and see what happens in the case where she does not have Corvo kill Daud. Such an act of using save data to "test out" mutually exclusive events in a video game’s possible narratives is a case of exploring the game’s possibility structure.

It makes no sense to attribute this sort of possibility-exploration to Corvo-and if we follow Robson and Meskin in identifying the player's fictional actions with those of the avatar, that seems to be the only way to explain this phenomenon as part of the game's narrative (ibid.).

The only other option for them, so far as I can see, is to dismiss such exploration as somehow "external to the narrative." They might argue that this kind of exploration is just like someone turning the pages of a book: the action is part of what it is to consume the narrative, but not part of the narrative itself. I grant that this is an available option for Robson and Meskin, but I do not see it as a particularly attractive one. The turning 
of a novel's pages does not affect what events will transpire next in the narrative; in contrast, exploration of a video game's possibility structure often directly influences which of the game's possible events end up constituting its actual narrative (as in the Dishonored example (ibid.)). When multiple events are fictionally realizable in a video game's possibility structure and one comes about rather than the other, it is reasonable to ask for a fictional explanation of what brought that one event about.

\section{The Epistemic Datum}

The epistemic datum is a datum about the nature of avatars: namely, despite the fact that the player apparently controls many aspects of the avatar of a video game, the avatar nonetheless (like any other character) has its own narratively significant beliefs, desires, and knowledge in which the player often does not share. Video game narratives often crucially depend on the player not sharing the avatar's set of beliefs, desires, and knowledge; a theory of avatar-player relations ought to be able to accommodate and explain this datum. The following example will help illustrate how video game narratives can deliberately separate avatar beliefs from player beliefs.

Spec Ops: The Line puts the player in control of Captain Martin Walker, the leader of a Delta Force team sent into a post-disaster Dubai to evacuate hostages (Yager Development 2012). As he proceeds through his mission with his team, he begins to go insane from the violence of war, hallucinating that a long-deceased Colonel is maliciously instigating insurgents and forcing Walker to do horrible things. The player, however, only discovers at the end of the narrative that Walker was insane: a different character ultimately reveals the truth about Walker's insane behavior.

A central aspect of this video game's narrative is that the player chooses to progress through the narrative despite it being obvious that her avatar is committing increasingly horrifying crimes against humanity: dousing refugees with white phosphorus, murdering other American soldiers, and so on (ibid.). It turns out that the avatar was insane, driven mad by war-but the player was not. The disturbing reality to which the game's fiction points is that the player soberly chose to continue playing the game, even as she was soberly aware of the avatar's wrongness. The player willfully enacted the actions of an insane killer.

Robson and Meskin cannot capture this core aspect of the narrative: you cannot capture the player's problematic decision to keep playing in the face of her avatar's atrocities by saying to the player, "You imagined that you were acting in justifiable ways, but it turns out that the person as whom you were imagining yourself (i.e. the avatar) was insane." Such an analysis fails to describe the dichotomy between the player's beliefs about her choices on the one hand, and the avatar's insane interpretation of those same 
events on the other hand: if fictional truths about the avatar are also fictional truths about the player, then it is either fictional that both of them are sane, or it is fictional that both of them are insane. ${ }^{7}$

Though Spec Ops is only a single case, the point generalizes: avatars are characters with fictional histories and mental lives, and the fact that a player can control some of the avatar's decisions does not entail that the player has access to these histories and mental lives (ibid.). That this lack of access can become narratively significant shows that a theory of video-game storytelling must accommodate and explain the general phenomenon.

\section{Grounding Narrative: The Possibility Structure and the Fictional Player}

Despite the initial attractiveness of Robson and Meskin's theory, the view faces substantial problems as an account of how avatars and players relate to the fictions of video games: it fails to accommodate and explain four central data about the medium it aims to analyze. I want to now suggest a very different way of approaching the player's role in video-game storytelling, which emphasizes the player's fictional metaphysical agency, rather than the player's relationship with the avatar.

We saw that we need to posit a new ontological entity in order to capture the fictions of video games: the possibility structure, which contains all possible events that can constitute the actual narrative within that game, together with relations that structure and govern the orders in which these possible events can be made actual. My proposal is that we take this possibility structure seriously as a new part of fiction, in the following sense: the possibility structure constitutes part of the fiction that functions as an abstract, metaphysically foundational space in the fiction, from which the game's possible narratives are derived. From this, it follows that the possible events in video games are really part of the video game's fiction even when they are merely possible: although such events are not part of the video game's actual narrative, they are part of the fictional metaphysical base that generates that actual narrative.

With that in mind, my proposal is that the narratives of video games are grounded in the operations of an abstract fictional entity - the fictional player-on the constituent possible events of this abstract fictional space-the possibility structure. The 'possible events' that this possibility structure contains are potentially actual events with conditional probabilities of being actualized. The fictional player acquires information about the organization of the possibility structure from the narrator, the entity that bridges the

7 It is true, of course, that there will not be a one-to-one correspondence between all avatar beliefs and all player (fictional) beliefs on Robson and Meskin's account. But the Spec Ops case captures something slightly different: it is a case in which the narrative centrally depends on a difference in the overall epistemic status of the player versus the avatar (sane vs. insane). I fail to see how this difference can be preserved and made narratively significant if the player is imagining herself as the avatar in Robson and Meskin's sense. 
video game's narrative and grounding metaphysics by expressing information about the possibility structure to the fictional player from within the narrative that the fictional player actualizes. ${ }^{8}$ The fictional player then operates on possible events of the possibility structure in such a way as to, in accordance with the events' conditional probabilities, make certain events obtain, thereby making a particular possible narrative actual. So, for example, the possibility structure of Dishonored contains the possible event $<$ Corvo killed Daud $>$ with probability $=1$ conditioned on the interaction of the fictional player with the event, and probability $=0$ otherwise (Arkane Studios 2012). ${ }^{9}$ Other possible events may have probabilities that prevent the fictional player from acting as an absolute determinant of that event: for example, Skyrim's possibility might contain the events $<$ The Dragonborn [i.e. the avatar] dealt $x$ damage to the dragon Alduin $>$, $<$ The Dragonborn dealt $y$ damage to the dragon Alduin $>$, and $<$ The Dragonborn dealt $z$ damage to the dragon Alduin>, each with probability 0.33 conditioned on the fictional player realizing the immediately preceding event $<$ The Dragonborn attacked the dragon Alduin> (Bethesda Game Studios 2011). Thus the possibility structure, as a fictional metaphysical foundation, establishes a well-defined set of possible narratives, all with well-defined probabilities of actually occurring; the fictional player is the entity that operates on the constituent possible events of the possibility structure, thereby updating the probabilities of these possible events in such a way as to establish the actual narrative of the video game, as identified with the player's playthrough. Taken together, it follows that the possibility structure and operations of the fictional player are the full, fictional ground for the video game's actual narrative.

The notion of an underlying fictional metaphysical structure grounding fictional worlds is substantially less intuitive than Robson and Meskin's theory, and so far as I can see it has no analogue in other narrative media: it uniquely emerges in video games as the best explanation of how players and developer-dictated possible events interact to make possible narratives in a video game actual.

No doubt, the fictional-player account of player interaction with video games has drawbacks. Most immediately, we are forced to accept that most ordinary language about video games is metaphorical: as players, we play the role of metaphysical actors, not avatars. Yet at the cost of giving up the theoretical accuracy of ordinary language,

8 An extended analysis of the narrating entity proposed here is warranted but beyond the scope of this article. Note, though, that the "narrator" of a video game is meant to be understood as a conceptual intermediary that expresses content from the game's possibility structure to the fictional player, similar to the kind of minimalist, conceptual narrator that George M. Wilson suggests exists in film $(2011,134)$. This narrating entity is not a character, nor is it the kind of voice-over narrative that sometimes features in film or video games: it is the much more foundational point of view through which an "audience" has access to a fictional world or set of possible fictional worlds. The use of the term 'narrator' is only intended to invoke a loose analogy between the entity I suggest here and more conventional narrating entities in other media, whereby both entities express the content of a fictional world to the some kind of metaphysically distinct audience, whether that audience is a flesh-andblood person or the fictional player.

$9 \quad$ I use these brackets to indicate sentences that represent events. 
we get a complete ontology of video games, subsuming both stochastic and non-stochastic events under a single analysis. Moreover, we now have the resources to offer illuminating, unified explanations of the explanatory, variability, exploratory, and epistemic data that are central to how video games function as a narrative medium.

\section{Explaining the Exploratory Datum}

Recall that Robson and Meskin's theory was unable to answer why, after much failing-and-repeating, the player's Skyrim avatar, Icarus, avoided a corner that had a monster hiding behind it (ibid.). With the fictional player, we have a natural answer: "The fictional player knew that there is a powerful monster behind that corner, and therefore made it the case that Icarus avoided that corner.”

More generally, the fictional-player account claims that there are two levels of explanation within the fiction of a video game: a world-internal level of explanation, and a grounding level of explanation. World-internal explanations are those that just reference facts about the constituents of a video game's actual narrative in the explanans. Grounding explanations, on the other hand, cite facts about the possibility structure and the fictional player in the explanans. For any given explanandum event in the actual narrative of a video game, both a world-internal explanation and a grounding explanation exist. What the monster-around-the-corner example shows is that the content of a video game's fictional world will sometimes underdetermine the world-internal explanation of an event: while the explanation presumably still exists, we do not have the resources from the video game's representational content to access the explanation. In such cases, therefore, we instead revert to the grounding explanation: the basis on which the fictional player realizes events through the possibility structure is always available to us, and it has particular explanatory value in cases like the monster around the corner because, in such cases, it is the only explanation accessible to us.

The fictional-player analysis is also explanatorily powerful here because it allows us to provide grounding explanations for a diversity of other fictional events that lack readily accessible world-internal explanations. Modern players of video games often read strategy guides to video games that outline the various areas in a video game's fiction, what can be found in these areas, and so on. A player of Skyrim might have read about the above monster-concealing corner in such a guide and therefore directed Icarus to avoid it; when asked the question of why Icarus avoided the corner, we can offer the same explanans as above: "The fictional player knew that there is a powerful monster behind that corner, and therefore made it the case that Icarus avoided it.” 
My analysis is also explanatorily powerful because it captures an equally relevant explanatory difference between the failing-and-repeating case and the strategy guide case. ${ }^{10}$ We can frame the difference in terms of a potential explanatory regress. Suppose that in both cases, after receiving the answer "The fictional player knew that there is a powerful monster behind that corner, and therefore made it the case that Icarus avoided it," our questioner persists: "How did the fictional player know about the monster?" In the failing-and-repeating case, we have a ready answer: namely, the process of failingand-repeating, by which the fictional player learned about the video game's possibility structure. Because this process is fully fictional-the fictional player explores fictional possibilities, and then fictionally realizes those possibilities-it is a proper and complete grounding explanation. But in the strategy guide case, it seems wrong to point to the strategy guide as part of the grounding explanation because the guide is not fictional in any sense. The fictional player did not read the guide: the real player did.

I think that it is right and informative to say, as the fictional-player view implies, that there simply is no further grounding explanation in the strategy guide case than "the fictional player simply knew.” For there is no further explanation for such cases within the fiction: the player stopped playing the role of fictional character in order to look for real-world sources of information about the video game. What is especially interesting here is that the case illuminates how the possibility structure of video games complicates the matter of players importing their real knowledge when engaging fiction: because video games' narrators (in most cases) only reveal a portion of video games' possibility structure to players at any given time as they engage the narrative, it is possible for the player to gain from external sources information about the possibility structure that they would not know simply by engaging the fiction for the first time on their own. This, I think, is the right account of why certain sources of a fictional player's knowledge - namely, those derived from the real player-are inadmissible as fictional explanations of the avatar's actions.

\section{Explaining the Variability Datum}

Our analysis of the fictional player as an agent that realizes fictional events explains what unifies video games' diversity of avatar relations. Return to the case of the avatarless wipEout, where Robson and Meskin were led to imply that the player imagines an avatar despite the lack of any such avatar explicitly represented in the game: we can instead say that, just like in video games with avatars, the fictional player is merely recognizing possible events within the fiction and making them actual (Psygnosis 1995). 
Further, the fictional player illuminates the connection between avatars and the narrative concept of point-of-view. Intuitively, there is clearly some relationship between avatars and the perspective through which a player experiences the fiction of a video game: even in video games where the player sees their avatar from some external perspective, rather than looking at the world of the video game through the avatar's eyes, the player is in some sense "tethered" to the avatar throughout the progression of the narrative. Can the fictional-player account offer a satisfying explanation of why players often seem tethered to avatars, given that there is no deep ontological connection between them?

My account can answer this challenge in a way that is contiguous with point-of-view in other media. The ways in which a player can actualize possible events within the fiction-i.e. the rules governing which events can be actualized in which order-tend to link actualizable events together in orders that maintain a coherent narrative focus. This focus may be on a single character (one avatar), a group of potential avatars, or some non-avatar entity (e.g., the racing craft in wipEout (ibid.)). The consistent focus affords narrative coherence: the quality of a narrative whereby its events collectively convey some kind of understanding to the audience.

I think that Velleman's notion of emotional cadence can help us get a grip on what sort of understanding a coherent narrative conveys. According to Velleman, emotions are sorted into natural sequences of arousal and subsequent resolutions - for example, "puzzlement [gives way to] curiosity, curiosity to foreboding, foreboding to horror, horror to grief-or perhaps instead to anger, which gives way to resentment, and so on" (Velleman 2003, 14). A coherent narrative, on this view, is one with events that are organized in such a way as to provide an audience with a complete emotional cadence: a sequence from arousal to resolution.

This model of narrative coherence allows us to explain why video games often focus on a single character, the avatar. It is easy to experience an emotional cadence-in any storytelling medium-by presenting the story through the point-of-view of a single character, with whose emotional journey the audience can sympathize or empathize. Video games can preserve this model of narrative coherence by making the locus of the fictional player's event-actualization be events concerning the actions of a particular character: designing the possibility structure in this way naturally invites the player to experience a full emotional cadence by emotionally investing in the game's avatar, which renders the video game's narrative coherent.

Note that none of the above entails that a video game structures its possibility space around an avatar to achieve narrative coherence. This is the right result: it allows us to account for video games that lack an avatar, as well as for video games that have multiple avatars. As an example from the former class of video game, consider Fire Emblem: 
rather than apparently following around and controlling a single character, the player of Fire Emblem (qua fictional player) plays the role of an army tactician: you have a god'seye view of a battlefield, and direct your troops to take various actions throughout the course of battle (Intelligent Systems 2003). ${ }^{11}$ Such a narrative can still achieve coherence, but it does not use a singular avatar to do so: instead, it makes the fictional player herself the focal character that unifies the narrative, following the emotional journeys of many different characters throughout the game. Thus our account gives us the flexibility to explain the multitudinous ways in which video games can achieve narrative coherence.

\section{Explaining the Epistemic Datum}

The fictional-player proposal is especially well equipped to explain the fact that the avatar's set of beliefs, desires, and knowledge can differ in narratively significant ways from the player's. Revisiting the narrative of Spec Ops: The Line, we now have the theoretical resources to fully capture the core of its narrative import: the fictional player, perfectly sane, comes to recognize that they are actualizing events in which Captain Walker commits terrible atrocities (Yager Development 2012). Walker is insane, driven mad by war, but the fictional player, aware of that insanity, nevertheless chooses to continue to enact events in which that insanity costs lives and insidiously aggravates Walker's PTSD.

The more we recognize the avatar as a character that is distinct from the fictional player, the better theoretical sense we can make of all sorts of video game stories. As it turns out, many video games capitalize on this sort of epistemic asymmetry between fictional player and avatar. Theoretically, this result was to be expected: given that the fictional player is making events the case with the focus on a character to which she has limited access (i.e. the avatar), it is as natural to tell stories that turn on the imperfection of that limited access as it is to tell stories with unreliable narrators, which generate a closely analogous sort of epistemic asymmetry between themselves and readers.

\section{Conclusion}

It is enticing to suppose that video games advance storytelling by furnishing players with more robust ways to inhabit the role of a character within their stories. Through my critique of Robson and Meskin's view, I have aimed to show that this approach to videogame storytelling — one that analyzes players as fictionally identical with avatars — is ultimately insufficient to accommodate and explain a wide range of central data about how video games tell stories. While I have not considered alternative extant accounts of

11 Note that some later games in the series (e.g., Fire Emblem: Awakening (Intelligent Systems and Nintendo SPD (2013))) do feature an avatar. These are not the entries in the series that I have in mind. 
player-avatar relations, I hope to have successfully made a case for why my proposed fictional-player analysis is promising and merits further research: when we approach the special storytelling of video games as a new, metaphysically robust way of representing possibility and ground in fiction, we gain fresh insight into what it is that makes video games an innovation in narrative form.

\section{Games}

Arkane Studios (2012). Dishonored. Xbox 360. Bethesda Softworks.

Bethesda Game Studios (2011). Skyrim. Xbox 360. Bethesda Softworks.

FromSoftware (2014). Dark Souls II. Xbox 360. Bandai Namco Games.

Intelligent Systems (2003). Fire Emblem. Nintendo. Game Boy Advance.

Intelligent Systems and Nintendo SPD (2013). Fire Emblem: Awakening. Nintendo. Nintendo 3DS.

Pajitnov, Alexey (1984). Tetris. Electronika 60.

Psygnosis (1995). WipEout. PlayStation.

Square (1997). Final Fantasy VII. Sony Computer Entertainment. PlayStation.

Yager Development (2012). Spec Ops: The Line. 2K Games. Xbox 360.

\section{References}

Aarseth, E. (2004). Quest Games as Post-Narrative Discourse. In Ryan (2004), 61-90.

Albert, T. \& Ramis, H. (1993). Groundhog Day. Columbia Pictures.

Bal, M. (2009). Narratology: Introduction to the Theory of Narrative. University of Toronto.

Bell, M. W. (2008). Toward a Definition of 'Virtual Worlds'. Journal of Virtual Worlds Research,

1(1). https://journals.tdl.org/jvwr/index.php/jvwr/article/viewFile/283/237. Accessed on 3/22/2017.

Bengson, J., Cuneo, T., and Shafer-Landau, R. (forthcoming). Methods, goals, and data in Moral

Theorizing. In The Routledge Handbook of Moral Epistemology (forthcoming).

Booth, W. C. (1983). The Rhetoric of Fiction. University of Chicago.

Juul, J. (2005a). Half-Real: Video Games Between Real Rules and Fictional Worlds. MIT.

(2005b). Half-Real: A Dictionary of Video Game Theory.

www.half-real.net/dictionary. Accessed 3/22/2017.

Klevjer, R. (2006). What is the Avatar? Fiction and Embodiment in Avatar-Based Singleplayer Computer Games (doctoral dissertation).

http://folk.uib.no/smkrk/docs/RuneKlevjer_What\%20is\%20the\%20Avatar_finalprint.pdf. Accessed 3/22/2017.

Leibniz, G. (1989). Philosophical Essays (Ariew, R. \& Garber, D., trans. \& eds.). Hackett.

Lewis, D. (1978). Truth in Fiction. American Philosophical Quarterly, 15(1), 37-46.

Murray, J. H. (1997). Hamlet on the Holodeck: The Future of Narrative in Cyberspace. Free.

Newman, J. (2002). The Myth of the Ergodic Videogame: Some Thoughts on Player-Character Relationships in Videogames. Game Studies: The International Journal of Computer Game Research, 2(1). 
http://www.gamestudies.org/0102/newman/. Accessed 3/22/2017.

Ney, A. (2013). Introduction. In Ney \& Albert (2013), 1-51.

Ney, A. \& Albert, D. (eds.) (2013). The Wave Function: Essays on the Metaphysics of Quantum Mechanics. Oxford University.

Reisz, K. (1966). The Technique of Film Editing. Focal.

Robson, J. \& Meskin, A. (2016). Video Games as Self-Involving Interactive Fictions. The Journal of Aesthetics and Art Criticism, 74(2), 165-177.

Ryan, M.-L. (ed.) (2004). Narrative Across Media: The Languages of Storytelling. University of Nebraska.

(2006). Avatars of Story. University of Minnesota.

Velleman, J. D. (2003). Narrative Explanation. The Philosophical Review, 112(1), 1-25.

Walton, K. L. (1990). Mimesis as Make-Believe: On the Foundations of the Representational Arts. Harvard University.

Wilson, G. M. (2011). Seeing Fictions in Film: The Epistemology of Movies. Oxford University.

Zimmerman, A., Jones, K., \& Timmons, M. (eds.) (forthcoming). The Routledge Handbook of Moral Epistemology. Routledge. 